\title{
Produção científica sobre atividade física e envelhecimento em programas brasileiros de pós- graduação em Educação Física
}

\author{
Giovane Pereira Balbé \\ Mauren da Silva Salin ${ }^{* *}$ \\ Giovana Zarpellon Mazo ${ }^{* * *}$ \\ Alexandro Andrade ${ }^{* * * *}$ \\ Ines Amanda Streit ${ }^{* * * * *}$
}

\begin{abstract}
Resumo: Este estudo investigou a produção científica sobre atividade física e envelhecimento em Programas de PósGraduação na área da Educação Física no Brasil. As informações originaram-se dos programas Stricto sensu em Educação Física, oriundos do banco de teses e dissertações da CAPES. Observou-se concentração dos trabalhos na região Sudeste, maior freqüência da pesquisa qualitativa e a entrevista como instrumento mais utilizado. Nos programas pioneiros, a concentração de grupos de pesquisa e a identificação com a temática em foco, de programas recentemente implantados, constituem-se nos aspectos que podem ter influenciado na produção científica voltados para a atividade física e envelhecimento.
\end{abstract}

Palavras-chave: Educação Física. Envelhecimento. PósGraduação. Produção Científica.

\footnotetext{
"Pesquisador voluntário do Laboratório de Atividade Física, Saúde e Envelhecimento - LAGER; Centro de Ciências da Saúde e do Esporte (CEFID); Universidade do Estado de Santa Catarina (UDESC) Florianópolis, SC, Brasil. e-mail: gbalbe@yahoo.com.br

"Doutoranda do Programa de Pós-Graduação em Ciências do Movimento Humano. Laboratório de Atividade Física, Saúde e Envelhecimento - LAGER; Centro de Ciências da Saúde e do Esporte (CEFID); Universidade do Estado de Santa Catarina (UDESC) Florianópolis, SC, Brasil.e-mail: mauren@softin.com.br

"'Professora do Programa de Pós-Graduação em Ciências do Movimento Humano. Coordenadora do Laboratório de Atividade Física, Saúde e Envelhecimento - LAGER; Centro de Ciências da Saúde e do Esporte (CEFID); Universidade do Estado de Santa Catarina (UDESC) Florianópolis, SC, Brasil e-mail: giovana.mazo@udesc.br

"...Professor do Programa de Pós-Graduação em Ciências do Movimento Humano. Coordenador do Laboratório de Psicologia do Esporte - LAPE; Centro de Ciências da Saúde e do Esporte (CEFID); Universidade do Estado de Santa Catarina (UDESC) Florianópolis, SC, Brasil. e-mail: alexandro.andrade@udesc.br

-..."Mestranda do Programa de Pós-Graduação em Ciências do Movimento Humano. Laboratório de Atividade Física, Saúde e Envelhecimento - LAGER, Centro de Ciências da Saúde e do Esporte (CEFID); Universidade do Estado de Santa Catarina (UDESC) Florianópolis, SC, Brasil.e-mail: inesamanda@gmail.com
} 


\section{INTRODUÇÃO}

O envelhecimento da população que vem ocorrendo em diferentes países, especialmente nos em desenvolvimento, é conseqüência da diminuição da mortalidade por doenças infectocontagiosas e do avanço tecnológico no tratamento das doenças crônico-degenerativas, associado à redução da fecundidade, permitindo uma maior expectativa de vida (COSTA, 2007).

Nessa perspectiva, o envelhecimento tornou-se foco de preocupação generalizada dos profissionais das mais variadas áreas, em especial a da saúde, em função da transição demográfica e epidemiológica observada a partir do início da década de 50 (GONÇALVES; BENEDETTI; MAZO, 2007). Entre os interesses, destaca-se o incentivo a prática de atividade física, em que a Educação Física tem contribuído de forma substancial.

O desenvolvimento de estratégias e políticas para manter e/ou aumentar a capacidade física em idosos, iniciou durante o IV Congresso Mundial de Atividade Física, Envelhecimento e Esporte em Heidelberg, Alemanha em 1996 (WHO, 1996), mas a partir de 2000 vários países e organizações publicaram diretrizes estruturadas e aplicáveis para o público idoso (GORZONI, 2007). Durante o evento, alguns pontos foram destacados, entre eles os benefícios imediatos e em longo prazo relativos a ganhos psicológicos, fisiológicos e sociais da atividade física e as contribuições científicas na área, porém com lacunas (WHO, 1996).

Gonçalves, Benedetti e Mazo (2007), com o objetivo de mapear o panorama atual dos grupos de pesquisa que estudam a atividade física e o envelhecimento humano no Brasil, encontraram 35 grupos de pesquisa cadastrados nessa temática. A maioria desses grupos está ligada às unidades de Instituições de Ensino Superior (IES), sendo que 15 desses são vinculados a programas de pós-graduação Stricto sensu, na área de Educação Física como também de áreas correlatas da saúde. Marzari e Acosta (2007) ao analisarem a 
produção científica da Educação Física e terceira idade, no período de 2001 e 2006, observaram o predomínio de estudos empíricos analíticos e o crescimento dos estudos de abordagem fenomenológica.

Considerando alguns estudos, os quais indicam que as poucas pesquisas na Educação Física estão voltadas a investigar produções monográficas (ASSIS; BONIFÁCIO, 2008; PAIVA; TAFFAREL, 2006) de cursos de graduação, além de abordagens específicas (SOUZA; LIMA; SILVA, 2004) ou gerais (SILVA, 2005), percebese que o interesse pela produção de conhecimento nesta temática é imprescindível, especialmente em relação às contribuições dos programas de pós-graduação em Educação Física.

Deste modo, o presente estudo representa uma iniciativa de buscar novos elementos que possibilitem investigar as contribuições científicas dessa temática, nos programas Stricto sensu em Educação Física no Brasil. Assim, se faz necessário realizar uma análise mais aprofundada e detalhada de quais aspectos são contemplados e quais necessitam de maior atenção nos estudos, favorecendo a construção de uma área mais sólida, consistente e fundamentada dos caminhos percorridos. Além disso, avaliar as contribuições dos programas de mestrado e doutorado, por meio de dissertações e teses possibilita ainda apontar lacunas e novos caminhos na busca de conhecimentos relevantes na área da atividade física e envelhecimento (GONÇALVES; BENEDETTI; MAZO, 2007).

Nesta perspectiva, este estudo objetivou investigar a produção científica sobre atividade física e envelhecimento nas dissertações e teses dos programas de pós-graduação em Educação Física no Brasil.

\section{MÉTOdo}

Trata-se de uma pesquisa documental e retrospectiva, apresentando um mapeamento da produção científica dos programas stricto sensu em Educação Física sobre a temática "Atividade Física e Envelhecimento", no período de 1996 a 2008. A busca ocorreu em 
teses e dissertações produzidas nos 21 programas de pós-graduação em Educação Física em nível de mestrado, sendo que 10 desses apresentam doutorado, cadastrados na CAPES (2009). A escolha deu-se pelos resumos dos trabalhos relacionados a Atividade Física e Envelhecimento, sendo esses selecionados no banco de dados da CAPES até o ano de 2008, data máxima disponibilizada pelo referido órgão no momento da pesquisa. A busca dos dados foi realizada no período de outubro e novembro de 2009.

Para a seleção das dissertações e teses, optou-se pela leitura dos resumos relacionados com a temática "Atividade Física e Envelhecimento", utilizando como critério de inclusão, a presença dos Descritores em Ciências da Saúde (DeCS): atividade física, idoso, envelhecimento, exercício físico e aptidão física. Tais descritores foram combinados aos pares, localizados por Instituição, utilizandose dos operadores lógicos disponíveis nas ferramentas de busca do banco de teses e dissertações da CAPES.

Desse modo, identificaram-se 749 resumos, sendo 483 resumos excluídos por não estarem vinculados a programas de pós-graduação Stricto sensu em Educação Física. Do mesmo modo, dos 266 resumos restantes, 144 também foram excluídos, uma vez que não se relacionaram à temática "atividade física e envelhecimento", restando, portanto, 122 resumos os quais foram analisados. Os critérios de seleção adotados encontram-se detalhados na figura 1 . 


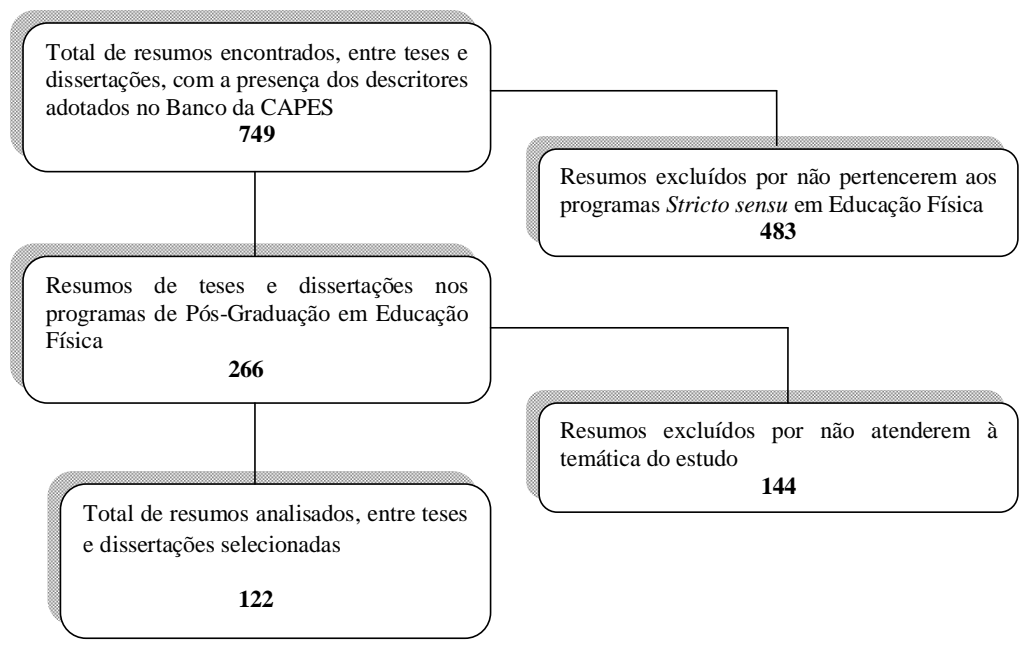

Figura 1 - Critérios de seleção dos resumos de teses e dissertações, referente a temática Atividade Física e Envelhecimento

Após a seleção dos 122 resumos entre teses e dissertações procedeu-se a leitura dos mesmos identificando as seguintes categorias: identificação (nível mestrado ou doutorado, Instituição, Unidade da Federação e ano de defesa), tipo de pesquisa e principais instrumentos, grupos amostrais e lócus de investigação.

Diante das categorias acima descritas, os dados foram organizados, tabulados e analisados no pacote estatístico Statistical Package for the Social Science (SPSS), versão 17.0 for Windows, mediante estatística descritiva simples (freqüência e percentual). A análise descritiva foi realizada por meio da frequiência de respostas observadas.

\section{Resultados}

Neste estudo foram encontrados 122 trabalhos, entre teses e dissertações, produzidos entre 1996 e 2008 pelos programas Stricto sensu em Educação Física nas Instituições de Ensino Superior (IES) brasileiras, abordando a temática Atividade Física e Envelhecimento. 
Dos trabalhos analisados, conforme apresenta a tabela 1, os descritores mais utilizados foram "atividade física", "idoso" e "envelhecimento".

Tabela 1 - Relação dos descritores utilizados nas teses e dissertações produzidas entre 1996 e 2008, abordando a temática Atividade Física e Envelhecimento

\begin{tabular}{lcc}
\hline \multicolumn{1}{c}{ Descritores } & Número de trabalhos & Percentual (\%) \\
\hline Atividade Física e Idoso & 56 & $45,9 \%$ \\
Atividade Física e Envelhecimento & 48 & $39,3 \%$ \\
Exercício Físico e Envelhecimento & 8 & $6,6 \%$ \\
Exercício Físico e Idoso & 6 & $4,9 \%$ \\
Aptidão Física e Idoso & 3 & $2,5 \%$ \\
Atividade Física e Terceira Idade & 1 & $0,8 \%$ \\
\hline
\end{tabular}

Em relação à distribuição da produção Stricto sensu brasileira em Educação Física Atividade Física e Envelhecimento (tabela 2) por região do país, constatou-se uma concentração de trabalhos na região sudeste $(60,7 \%)$ seguido da região sul $(25,4 \%)$ e da região centro-oeste $(13,9 \%)$. Ao observar a produção encontrou-se uma concentração de trabalhos no Estado de São Paulo (52,5\%), seguido de Santa Catarina (21,4\%), Distrito Federal $(13,9 \%)$, Rio de Janeiro $(7,4 \%)$, Paraná $(2,4 \%)$, Rio Grande do Sul $(1,6 \%)$ e Minas Gerais $(0,8 \%)$. Não foram observados estudos, na temática pesquisada, nos demais estados brasileiros. Dos 21 programas, 9 (42,9\%) não apresentaram estudos referentes a temática pesquisada, sendo esses pertencentes as Universidades: Cruzeiro do Sul (UNICSUL), Salgado de Oliveira (UNIVERSO), de Brasília (UNB), Federal de Viçosa (UFV), Federal do Paraná (UFPR), Federal de Pelotas (UFPEL), Estadual de Londrina (UEL), Federal do Espírito Santo (UFES) e Fundação Universidade de Pernambuco (FESP). 
Tabela 2 - Distribuição das IES por Unidade da Federação (UF), Programa de PósGraduação (PPG), ano de criação dos mesmos e número de teses e dissertações produzidas na temática Atividade Física e Envelhecimento

\begin{tabular}{|c|c|c|c|c|}
\hline \multirow{2}{*}{ UF/IES } & \multirow{2}{*}{ PPG } & \multirow{2}{*}{ Ano de Criação } & \multicolumn{2}{|c|}{$\begin{array}{c}\mathrm{N}^{0} \text { de Teses e } \\
\text { Dissertações }\end{array}$} \\
\hline & & & $\begin{array}{l}\text { Frequência } \\
\qquad(f)^{*}\end{array}$ & $\begin{array}{c}\text { Percentual } \\
(\%)\end{array}$ \\
\hline \multicolumn{5}{|l|}{ São Paulo } \\
\hline UNESP & $\mathrm{M} / \mathrm{D}$ & $1991 / 2001$ & 16 & 13,1 \\
\hline UNICAMP & $\mathrm{M} / \mathrm{D}$ & $1988 / 1993$ & 15 & 12,3 \\
\hline UNIMEP & $\mathrm{M}$ & 2000 & 13 & 10,7 \\
\hline USP & $\mathrm{M} / \mathrm{D}$ & $1977 / 1989$ & 13 & 10,7 \\
\hline USJT & M & 2004 & 7 & 5,7 \\
\hline UNICSUL & M & 2007 & - & - \\
\hline \multicolumn{5}{|c|}{ Santa Catarina } \\
\hline UFSC & $\mathrm{M} / \mathrm{D}$ & $1996 / 2006$ & 15 & 12,3 \\
\hline UDESC & $\mathrm{M} / \mathrm{D}$ & 1997/2009 & 11 & 9,1 \\
\hline \multicolumn{5}{|c|}{ Distrito Federal } \\
\hline UCB & $\mathrm{M} / \mathrm{D}$ & $1999 / 2006$ & 17 & 13,9 \\
\hline UNB & M & 2006 & - & - \\
\hline \multicolumn{5}{|c|}{ Rio de Janeiro } \\
\hline UGF & $\mathrm{M} / \mathrm{D}$ & 1985/1994 & 9 & 7,4 \\
\hline UNIVERSO & M & 2006 & - & - \\
\hline UFRJ & M & $\mathrm{AH}$ & - & - \\
\hline \multicolumn{5}{|l|}{ Paraná } \\
\hline UFPR & $\mathrm{M} / \mathrm{D}$ & $2002 / 2007$ & 3 & 2,4 \\
\hline UEL & M & 2006 & - & - \\
\hline \multicolumn{5}{|c|}{ Rio Grande do Sul } \\
\hline UFRGS & $\mathrm{M} / \mathrm{D}$ & 1989/1999 & 2 & 1,6 \\
\hline UFPEL & M & 2007 & - & - \\
\hline \multicolumn{5}{|c|}{ Minas Gerais } \\
\hline UFMG & $\mathrm{M} / \mathrm{D}$ & $1989 / 2008$ & 1 & 0,8 \\
\hline UFV & M & 2007 & - & - \\
\hline \multicolumn{5}{|c|}{ Espírito Santo } \\
\hline UFES & M & 2006 & - & - \\
\hline \multicolumn{5}{|l|}{ Pernambuco } \\
\hline FESP & M & 2008 & - & - \\
\hline Total & $21 / 10$ & & 122 & $100 \%$ \\
\hline
\end{tabular}

*A ordem de apresentação das instituições foi decrescente, conforme a frequência de teses e dissertações produzidas nos referidos programas; M - Mestrado; D Doutorado; UNIVERSO - Universidade Salgado de Oliveira; UNESP - Universidade Estadual Paulista Júlio de Mesquita Filho; UFMG - Universidade Federal de Minas Gerais; UFRGS - Universidade Federal do Rio Grande do Sul; UDESC - Universidade do Estado de Santa Catarina; UNICSUL - Universidade Cruzeiro do Sul; UNB Universidade de Brasília; UCB - Universidade Católica de Brasília; UFES - 
Universidade Federal do Espírito Santo; UFV - Universidade Federal de Viçosa; UFPR - Universidade Federal do Paraná; UFRJ - Universidade Federal do Rio de Janeiro; UGF - Universidade Gama Filho; UFPEL - Universidade Federal de Pelotas; UFSC Universidade Federal de Santa Catarina; USP - Universidade de São Paulo;

UNICAMP - Universidade de Campinas; UNIMEP - Universidade Metodista de

Piracicaba; USJT - Universidade São Judas Tadeu; FESP - Fundação Universidade de Pernambuco; UEL - Universidade Estadual de Londrina. AH - Aguardando

Homologação pelo Conselho Nacional de Educação.

Observa-se que dos 122 trabalhos produzidos, 118 são dissertações de mestrado e apenas 4 são teses de doutorado (figura 2). As primeiras dissertações, representando 15,6\% dos trabalhos $(\mathrm{n}=19)$, foram defendidas no período de 1996 a 2001. Entre 2002 e 2004, constata-se um crescimento de $84,2 \%$ no número de dissertações defendidas, em relação ao início do período analisado, e, entre 2005 e 2008 esse aumento foi de 82,9\%. Quanto às 4 teses, constatou-se que as mesmas foram defendidas entre 2004 e 2008, sendo que as duas primeiras defendidas foram na Universidade Gama Filho (UGF), uma em 2004 e outra em 2006. As outras duas foram defendidas na Universidade de Campinas, nos anos de 2006 e 2008.

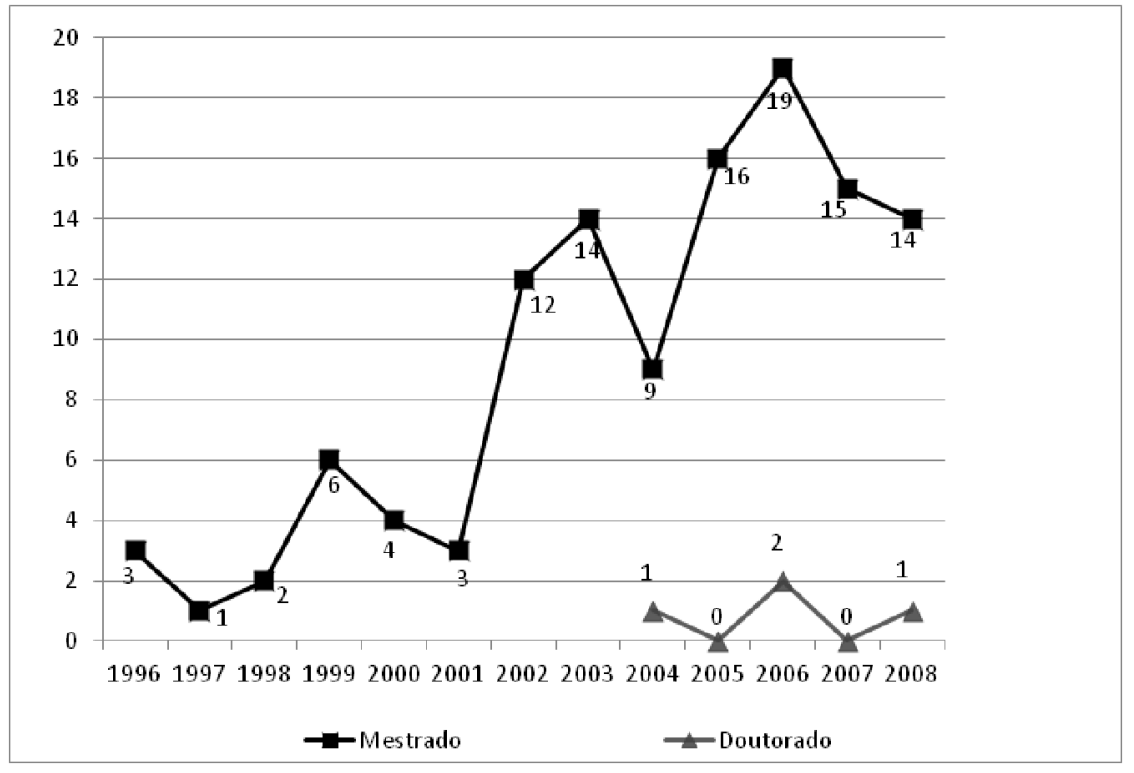

Figura 2 - Resultado da análise documental de dissertações e teses produzidos pelos programas de Stricto sensu em Educação Física no Brasil, refente a temática Atividade Física e Envelhecimento, no período de 1996 - 2008. 
Quanto ao tipo de pesquisa, observou-se que $14,7 \%$ dos trabalhos são de cunho qualitativo e $12,3 \%$ descritivo. No entanto, não foi possível indentificar 58,2\% dos trabalhos analisados (figura $3)$.

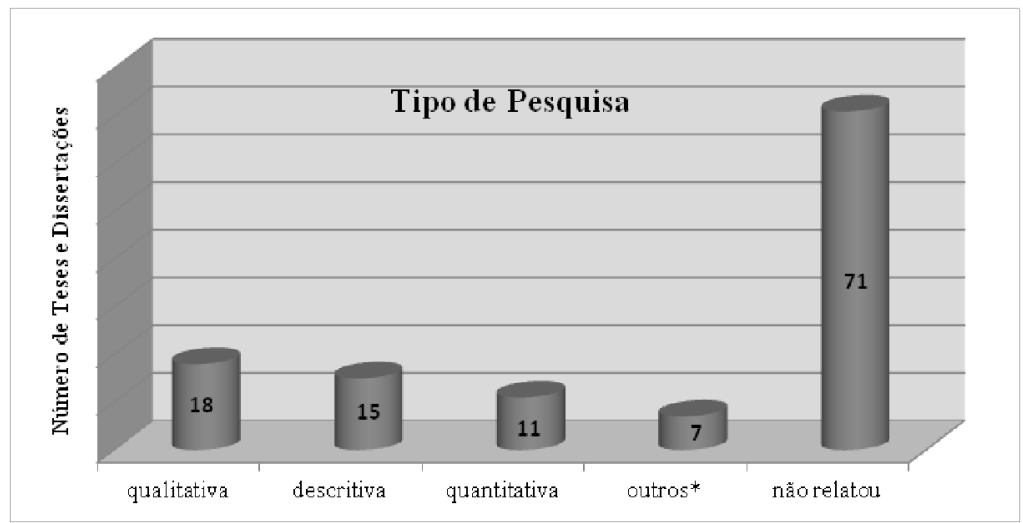

Figura 3. Número de teses e dissertações conforme o tipo de pesquisa produzidos pelos programas de Stricto sensu em Educação Física no Brasil, refente a atividade física e envelhecimento.

* Pesquisas de campo, quali-quantitativa, bibliográfica e sócio-pedagógica

Em relação aos instrumentos de pesquisa constatou-se que 14,6\% foram entrevistas, 7,3\% utilizaram o Questionário Internacional de Atividade Física (IPAQ) e 6,7\% adotaram um questionário semiestruturado. Além disso, identificou-se uma diversidade de instrumentos, os quais foram categorizados como outros $(52,2 \%)$, pois os mesmos foram utilizados no máximo em três trabalhos, entre eles destaca-se a bateria da American Alliance for Health, Physical Education, Recreation and Dance (AAHPERD), Mini-Exame do Estado Mental (MEEM) e Escala de Depressão Geriátrica (GDS), entre outros, conforme tabela 3. 
Tabela 3 - Frequência e percentual dos principais instrumentos utilizados nas teses e dissertações pesquisadas.

\begin{tabular}{lcc}
\hline Variáveis & $\begin{array}{c}\text { Frequência } \\
(\boldsymbol{f})\end{array}$ & $\begin{array}{c}\text { Percentual } \\
(\boldsymbol{\%})\end{array}$ \\
\hline Instrumentos & 26 & 14,6 \\
Entrevista & 13 & 7,3 \\
IPAQ & 12 & 6,7 \\
Questionário Semi-estruturado & 6 & 3,4 \\
Teste 1RM & 6 & 3,4 \\
Observação & 4 & 2,2 \\
Questionário de Baecke & 4 & 2,2 \\
Dinamômetro & 3 & 1,7 \\
AAHPERD & 3 & 1,7 \\
MEEM & 3 & 1,7 \\
GDS & 3 & 1,7 \\
Bateria de Andreotti \& Okuma & 3 & 1,7 \\
Densidometria & 22 & 12,4 \\
Não relatou & 70 & 52,2 \\
Outros* & & \\
\hline
\end{tabular}

*Teste de 1 milha, Whogol-bref, Teste de 6 minutos, etc.; IPAQ - Questionário Internacional de Atividade Física; Teste 1RM - Teste de repetição máxima;

AAHPERD - Bateria de Testes Motores da American Aliance for Health, Physical Education, Recreation and Dance; MEEM - Mini-Exame do Estado Mental; GDS Escala de Depressão Geriátrica.

Entre as categorias, quanto às características amostrais, identificou-se o foco em amostras constituídas de idosos ativos e sedentários. Por outro lado, se observa o interesse em estudos com idosos longevos, sendo uma tentativa de melhor entender a influência da atividade física no envelhecimento em idades mais avançadas, bem como em grupos especiais, tais como em hipertensos, cardiopatas, parkinsonianos, entre outros (tabela 4). Ao observar a tabela 4, quanto aos locais em que os pesquisados encontravam-se inseridos, constatou-se um predomínio nos programas de atividade física. 
Tabela 4 - Frequência e percentual das características amostrais e lócus de investigação destacados nas teses e dissertações pesquisadas.

\begin{tabular}{lcc}
\hline Características Amostrais & $\begin{array}{c}\text { Frequência } \\
(f)\end{array}$ & $\begin{array}{c}\text { Percentual } \\
(\boldsymbol{\%})\end{array}$ \\
\hline Idosos Ativos & 31 & 25,4 \\
Idosos Sedentários & 19 & 15,6 \\
Grupos Especiais* & 10 & 8,2 \\
Longevos & 3 & 2,4 \\
Não relatou & 59 & 48,4 \\
\hline Lócus de Investigação & & \\
\hline Programas de Atividade Física & 20 & 16,4 \\
Centros de Convivência & 7 & 5,7 \\
Instituições de Longa Permanência & 7 & 5,7 \\
Projeto Extensão/Universidade 3a Idade & 5 & 4,1 \\
Não relatou & 83 & 68 \\
\hline
\end{tabular}

*Jovens, Adultos, Parkinsonianos, Cardiopatas, Hipertensos, Cegos ou Pósmenopausa;

\section{Discussão}

$\mathrm{O}$ crescente interesse e reconhecimento da atividade física para idosos, no intuito de preservar suas capacidades funcionais e reduzir fragilidades, têm provocado o surgimento de equipes e instalações voltadas ao trabalho a essa população (GORZONI, 2007). Esta inferência vem ao encontro dos resultados apresentados pelo presente estudo, em que a produção científica dos programas de mestrado e doutorado em Educação Física aponta a atividade física e o idoso como os principais focos de estudo abordados.

A centralização da produção científica na área investigada, no Estado de São Paulo, assim como o fato de Santa Catarina se destacar como a segunda Unidade da Federação com maior produção nos programas de Educação Física voltados a Atividade Física e Envelhecimento e a não produção de teses e dissertações em 9 dos 21 programas investigados, refletem três aspectos relevantes na produção científica dos programas Stricto sensu da Educação Física brasileira. Primeiro, o pioneirismo dos programas na área favorece para a maior produção; segundo, o surgimento de grupos de pesquisa direcionados ao trabalho científico na Gerontologia, em especial na Atividade Física e Envelhecimento, como acontece nas Instituições de Ensino Superior UDESC e UFSC e, terceiro, a recente criação 
de programas de pós-graduação na Educação Física, também parece ser um forte indicativo de influência na produção científica brasileira quanto a Atividade Física e Envelhecimento. Nesta perspectiva, Coutinho et al. (2009), corroboram os dados ao apontar que, principalmente, a partir do ano de 2000 as regiões sudeste e sul são os principais centros de produção de conhecimento sobre o tema em relação a área da Educação Física.

Outro aspecto proeminente no presente estudo refere-se à baixa produção de teses de doutorado pelos programas de doutorado em Educação Física na temática em estudo, refletindo o reduzido número de doutores/orientadores na Educação Física direcionados no trabalho da Atividade Física e Envelhecimento. Este fato pode ser observado nas quatro teses encontradas nesta investigação, denotando a falta de orientadores especializados, o que demanda a necessidade de formação de doutores em Educação Física com interesse nessa temática.

Neste panorama, os estudos da Atividade Física e Envelhecimento são ainda recentes, observando-se as primeiras dissertações defendidas na Educação Física a partir de 1996, com um elevado interesse nos anos de 2002 e 2003. As primeiras dissertações foram produzidas nos programas das IES consolidadas, ou seja, os primeiros programas de mestrado em Educação Física no Brasil. Gorzoni (2007) explica que o desenvolvimento de estratégias e políticas para manter e/ou aumentar a capacidade física em idosos, iniciado durante o IV Congresso Mundial de Atividade Física, Envelhecimento e Esporte em Heidelberg, Alemanha em 1996, foi relevante para que, a partir de 2000, vários países e organizações publicassem diretrizes estruturadas e aplicáveis para o público idoso.

No Brasil, esse fato pode estar conexo ao incremento quantitativo na produção científica, em primeiro lugar, a partir da década de 90, com a expansão das universidades de terceira idade e também com a formação de grupos de pesquisa (GOLDSTEIN, 2002) e, em outro momento, coincidindo com a criação de cursos de Pós-Graduação em Gerontologia, a partir de 2003 (MARZARI; 
ACOSTA, 2007). Ao verificar os locais de inserção dos pesquisados, o presente estudo averigua um predomínio nos programas de atividade física, mostrando o papel e a necessidade da organização desses no incentivo a prática e orientação para um envelhecimento ativo.

O florescimento de grupos de pesquisa a partir de 2002 demonstra o interesse pelo estudo da atividade física na saúde das pessoas idosas, conforme identificado no estudo de Gonçalves, Benedetti e Mazo (2007), que, ao realizarem um levantamento dos grupos de pesquisa na área, encontraram um total de 35 grupos. Segundo as autoras a maioria dos estudos sobre essa temática encontra-se vinculada aos grupos de pesquisa inseridos nas unidades de Instituições de Ensino Superior (IES), confirmando que em nosso país, as atividades de pesquisa têm sido realizadas, mormente por docentes universitários. Nesta perspectiva, a ativação e agregação da pós-graduação a núcleos de investigação, determinam um maior relacionamento com as atividades de laboratório, tornando-se primordial, uma vez que "sem pesquisa não há pós-graduação" (AMADIO, 1999).

Nesse aspecto, devido este estudo estar focado nas contribuições dos programas de pós-graduação Stricto sensu em Educação Física e por não considerar os grupos de pesquisa vinculados, caracterizando-se como um fator limitador, persistem lacunas, haja vista que os grupos de pesquisa podem influenciar na construção de teses e dissertações na área em estudo.

Quanto aos aspectos metodológicos investigados, observou-se a prevalência de pesquisa de cunho qualitativo e descritivo, que segundo Alves (2009), representa uma nova alternativa nas pesquisas em Educação Física. Destaca-se, no entanto, que 58,2\% dos trabalhos analisados não foram identificados quanto ao tipo de pesquisa, chamando a atenção para a falta de padronização nos resumos disponíveis pela CAPES. Esse fato restringe a análise, o aprofundamento e detalhamento dos aspectos metodológicos desenvolvidos pelos autores, desfavorecendo a difusão da produção de conhecimento dos programas Stricto sensu em Educação Física 
voltados a Atividade Física e Envelhecimento. Assim, a falta de detalhamento dos aspectos metodológicos de inúmeros resumos entre os trabalhos analisados desfavorece as suas reais contribuições para o progresso científico, já que as avaliações sistemáticas da produção e do trabalho dos pesquisadores garantem o aperfeiçoamento constante não só do conhecimento como também do próprio ensino.

Portanto, destaca-se a necessidade de um maior controle pelo setor responsável da CAPES ao promover o banco de dados, devendo antes, analisar a estrutura dos resumos por ela disponibilizados. Tal iniciativa irá favorecer que os trabalhos desta natureza sejam aperfeiçoados e aprofundados. Salienta-se ainda que, tais resultados devem ser interpretados com precaução, pois essa falta de informação não propicia um diagnóstico real do tipo de pesquisa utilizado em teses e dissertações produzidos nos programas ora analisados.

Estudo abrangendo a tríade: grupo de pesquisa, teses e dissertações e artigos científicos, objetivou analisar a pesquisa na temática "Envelhecimento" desenvolvido pela Educação Física de 1997 a 2007 (COUTINHO et al., 2009) Nesta investigação, dos programas de Mestrado e Doutorado constantes da lista de cursos recomendados pela CAPES, os autores colocam que dos 20 cursos, apenas 12 apresentaram o conteúdo das teses e dissertações disponíveis on-line. Esta dificuldade foi colocada também por Goldstein (2002), no estudo sobre a produção científica na área da Gerontologia, onde avalia o sistema de rastreamento de informações como carentes, além da falta de unificação das palavras-chave usadas na área. Amadio (1999) destaca entre as perspectivas e tendências da pós-graduação a necessidade de se disseminar a comunicação e divulgar documentos eletrônicos, através da democratização das redes universitárias.

Identificou-se uma pluralidade de instrumentos utilizados nos estudos de dissertações e teses investigados, demonstrando o quanto os estudos voltados à área de Atividade Física e Envelhecimento são recentes e dispersos. Essa constatação pode refletir em dois aspectos: a dificuldade para aprofundamento na área e menores 
condições de comparação entre estudos realizados no Brasil. Goldstein (2002) corrobora com essa idéia colocando que a falta de articulação entre os diferentes programas e bibliotecas mantém a produção acadêmica na obscuridade e, desta forma o aproveitamento do conhecimento fica minimizado.

\section{Considerações FINAIS}

Contemplando o objetivo proposto, este estudo apresenta resultados sobre a produção científica em programas brasileiros de pós-graduação na temática atividade física e envelhecimento. $\mathrm{O}$ aumento no número de teses e dissertações que ocorre a partir do ano 2000, principalmente na região sudeste e sul do Brasil coincide com a formação de grupos de pesquisa e criação de cursos de pósgraduação em gerontologia.

Os programas pioneiros, a possível concentração de grupos de pesquisa e a identificação de programas recém implantados caracterizam-se como aspectos que podem ter influenciado na produção científica nos programas de pós-graduação Stricto sensu em Educação Física, voltados à temática Atividade Física e Envelhecimento.

Ao final da busca, alguns trabalhos podem não ter sido localizados, fato que pode ser atribuído ao não lançamento destes no correspondente acervo virtual. Nota-se que o sistema de rastreamento da informação ainda é carente e o aumento da produção científica necessita a adoção de medidas que conduza sua divulgação, já que a avaliação sistemática dos trabalhos produzidos contribui para aprimorar o conhecimento, além de apontar lacunas e atender às demandas da sociedade, sobretudo do público idoso.

Considerando as limitações do estudo, algumas recomendações poderiam ser feitas para investigações futuras. Independente de ter linhas de pesquisa específicas ou não, corresponde ao primeiro passo para o aprofundamento da próxima busca, a partir da base do Diretório de Grupos de Pesquisa/CNPq, identificar outras fontes 
envolvidas, como o currículo Lattes dos pesquisadores, o DATA/ CAPES acerca dos programas de mestrado e doutorado com suas áreas de concentração, linhas de pesquisa e produção de dissertações e teses. 


Scientific researches about the connection
between physical exercises and aging in
Brazilian programs of postgraduation in
Physical Education
Abstract: This study aimed to analyze some scientific
researches about the connection between physical
exercises and aging in Brazilian programs of graduate
in Physical Education. The information came from the
graduate studies program in Physical Education, from
the bank of theses and dissertations from CAPES.
Observed concentration of jobs in the Southeast, more
frequently and qualitative research interview as the
most used tool. Inpioneering programs, the
concentration of research groups and identification
with the subject in focus, to newly established
programs, are on the aspects that may have influenced
the scientific-oriented physical activity and aging.
Key words: Physical Education. Aging.
Postgraduation. Scientific Research.

\section{Producción científica sobre actividad física y envejecimiento en programas brasileños de postgrado en Educación Física}

Resumen: Este estudio investigó la producción científica sobre actividad física y envejecimiento en Programas de Postgrado en el área de la Educación Física en Brasil. Las informaciones son originarias de los programas stricto sensu en Educación Física, oriundos del banco de tesis y disertaciones de la CAPES. Se observó una concentración de trabajos en la región Sudeste, una mayor frecuencia de investigación cualitativa y la entrevista como el instrumento más utilizado. En los programas pioneros, la concentración de grupos de investigación y la identificación con la temática en foco, de programas recién implantados, se constituyen en los aspectos que pueden haber influenciado la producción científica enfatizando la actividad física y el envejecimiento.

Palabras clave: Educación Física. Envejecimiento. Postgrado. Producción Científica. 


\section{REFERÊNCIAS}

ALVES, F. S. Pesquisa qualitativa: desbravando territórios possíveis de se fazer ciência na Educação Física. Motriz, Rio Claro, v. 15, n. 4, p. 955-963, out./dez. 2009.

AMADIO, A. C. Os caminhos da pós-graduação "Stricto Sensu". Revista Paulista de Educação Física, São Paulo, v. 13, n. esp., p. 36-41, dez. 1999.

ASSIS, R. M.; BONIFÁCIO, N. A. A produção científica na formação dos alunos do curso de educação física do CAJ/UFG. Revista Eletrônica Itinerarius Reflectionis, Goiás, v. 2, n. 5, p. 1-10, jul./dez. 2008.

COORDENAÇÃO DE APERFEIÇOAMENTO DE PESSOAL DE NÍVEL SUPERIOR CAPES. Banco de Teses. 2009. Disponível em: <http://www.capes.gov.br/capes/ portal.htm>. Acesso em: 12 out. 2009.

COSTA, J. L. R. Novas perspectivas de políticas na área da atividade física e envelhecimento. Revista Brasileira de Cineantropometria e Desempenho Humano, v. 9, supl. 1, p. s15-s20, maio 2007.

COUTINHO, R. X. et al. Análise da pesquisa da Educação Física na temática envelhecimento humano. Lecturas Educación Física y Desportes, Buenos Aires, v. 14, p. 1- 1, 2009.

GOLDSTEIN, L. A produção científica na área da gerontologia. 2002. Disponível em: <http: //www.comciencia.br/reportagens/envelhecimento/texto/env17.htm>. Acesso em: 29 nov. 2010.

GONÇALVES, L. H. T.; BENEDETTI, T. R. B.; MAZO, G. Z. Pesquisa e avanços científicos na área de atividade física e envelhecimento. Revista Brasileira de Cineantropometria e Desempenho Humano, v. 9, supl. 1, p. s57-s60, maio 2007.

GORZONI, M. L. Programas de atividade física e políticas públicas internacionais. Revista Brasileira de Cineantropometria e Desempenho Humano, v. 9, supl. 1, p. s13-s14, maio 2007.

MARZARI, J.; ACOSTA, M. A. F. Diagnóstico da produção científica na temática terceira idade no período 2001-2006. Revista Brasileira de Ciências do Esporte, v. 29 , n. 1 , p. 123-141, maio 2007.

PAIVA, A. C.; TAFFAREL, C. N. Z. Produção científica na graduação: desafios para a educação física. In: REUNIÃO ANUAL DA SBPC. 58., 2006, Florianópolis. Anais... Disponível em: <http://www.sbpcnet.org.br/livro/58ra/SENIOR/RESUMOS/ resumo_3302.html>. Acesso em: 13 out. 2009. 
SILVA, R. V. S. A história da produção científica em educação física no Brasil a partir das teses e dissertações. In: CONGRESSO DE CIÊNCIA DO DESPORTO, 1. 2005, Campinas. Disponível em: <http://www.fef.unicamp.br/hotsites/ccd_2005/ cd/trabalhos/mesa/ROSSANA\%20V.S.pdf>. Acesso em 13 out. 2009.

SOUZA, J. A.; LIMA, L. F.; SILVA, R. V. S. A história oral nas teses e dissertações em educação física. In: SEMINÁRIO INTERNACIONAL DE PESQUISA E ESTUDOS QUALITATIVOS: A PESQUISA QUALITATIVA EM DEBATE, 2., Bauru, 2004, Baurú/ SP. Anais... Bauru/SP: Editora da Universidade do Sagrado Coração, 2004.

WORLD HEALTH ORGANIZATION - WHO. The Heidelberg guidelines for promoting physical activity among older persons. 1996. Disponível em: <http://www.uwo.ca/ actage/proceedings/>. Acesso em: 13 out. 2009.

Endereço para Correspondência:

Laboratório de Atividade Física, Saúde e Envelhecimento - LAGER;

Centro de Ciências da Saúde e do Esporte (CEFID);

Universidade do Estado de Santa Catarina (UDESC)

Rua Pascoal Simone, 358. Bairro Coqueiros.

CEP: 88080-350 Florianópolis, SC, Brasil.

e-mail: mauren@softin.com.br

Recebido em: 19.04.2011

Aprovado em: 27.03.2012

Vovimento, Porto Alegre, v. 18, n. 01, p. 261-279, jan/mar de 2012. 
